\title{
Matrix metalloproteinase expression patterns in luminal A type breast carcinomas
}

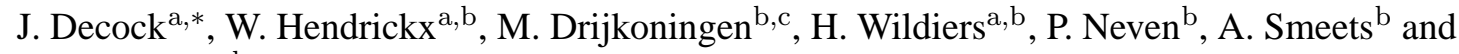 \\ R. Paridaens ${ }^{a, b}$ \\ ${ }^{a}$ Laboratory for Experimental Oncology (LEO), Department of General Medical Oncology, University Hospital \\ Gasthuisberg, Herestraat 49, 3000 Leuven, Belgium \\ ${ }^{\mathrm{b}}$ Multidisciplinary Breast Center, University Hospital Gasthuisberg, Herestraat 49, 3000 Leuven, Belgium \\ ${ }^{c}$ Pathology Department, University Hospital Sint Rafaël, Minderbroedersstraat 12, 3000 Leuven, Belgium
}

\begin{abstract}
Objective: Aberrant expression of individual matrix metalloproteinases has been associated with poor prognosis in various human carcinomas. The current study aimed at defining an RNA expression profile of various MMPs in breast cancer and correlating their expression with clinicopathological parameters.

Methods: The RNA expression patterns of 6 MMPs (MMP2, MMP8, MMP9, MMP10, MMP11, MMP13) were determined in 25 breast carcinomas using quantitative RT-PCR and correlated with clinicopathological parameters, including menopausal status, tumor size and grade, and lymph node involvement.

Results: We observed high MMP2 levels more frequently in premenopausal than in postmenopausal women $(p=0.02)$. Analysis of luminal A type invasive ductal carcinomas (19/25), revealed an even stronger association of MMP2 with menopausal status ( $p=$ 0.005). Within this subgroup, we also found a correlation between MMP11 and menopausal status $(p=0.02)$. No correlation was found between MMP expressions and other clinicopathological parameters. In co-expression analyses MMP2-MMP10 and MMP8-MMP9 showed a weak correlation of their expression.

Conclusions: Although this is a pilot study, our findings indicate that luminal A invasive ductal carcinomas commonly express high MMP2 and MMP11 levels in premenopausal breast cancer patients and suggest a co-regulation of MMP2-MMP10 and MMP8-MMP9.
\end{abstract}

Keywords: Breast cancer, matrix metalloproteinases, menopausal status, luminal A, clinicopathological parameters

\section{Introduction}

Breast cancer is one of the most common cancers in the world. Despite early diagnosis and administration of adjuvant systemic therapy, metastasis accounts for the majority of treatment failures and deaths. The classical prognostic parameters of breast carcinoma include tumor size and grade, axillary lymph node involvement, menopausal status, steroid hormone receptor and Her2/neu status. However, prognostic strat-

\footnotetext{
* Corresponding author: J. Decock, Lab for Experimental Oncology, University Hospital Gasthuisberg, O\&N 1, Room 815, Herestraa 49, B-3000 Leuven, Belgium. Tel.: +32 163462 93; Fax: +32 16 3469 01; E-mail: julie.decock@med.kuleuven.be.
}

ification of patients according to these clinicopathological parameters fails to identify all patients at risk as illustrated by heterogeneous clinical outcome. Recent DNA microarray profiling studies on breast tumors have identified distinct subtypes of breast carcinomas that are associated with different clinical outcomes [1, 17,18]. Using an intrinsic set of 534 genes, Sorlie et al. analyzed the expression profiles of 115 independent locally advanced breast tumor samples and categorized them into five groups according to their estrogen receptor (ER), progesterone receptor (PR) and human epidermal growth factor receptor (Her2/neu) expression: luminal $\mathrm{A}\left(\mathrm{ER}^{+} / \mathrm{PR}^{+-} / \mathrm{Her} 2^{-}\right)$, luminal $\mathrm{B}$ $\left(\mathrm{ER}^{+} / \mathrm{PR}^{+-} / \mathrm{Her} 2^{+}\right)$, normal breast-like, Her2 ${ }^{+} / \mathrm{ER}-$ $\left(\mathrm{ER}^{-} / \mathrm{PR}^{-} / \mathrm{Her} 2^{+}\right)$and basal-like $\left(\mathrm{ER}^{-} / \mathrm{PR}^{-} / \mathrm{Her} 2^{-}\right)$ 
with the latter two associated with poor outcomes [18]. Classification of breast tumors into these distinct subtypes in combination with classical clinicopathological parameters might improve prognostic accuracy. Furthermore, identification of new biomarkers of invasion and metastasis might result in a better prognostic stratification and lead to the development of specific therapies directed towards the prevention of invasion.

Tumor invasion and metastasis are the result of a multi-step process that includes basement membrane disruption, stromal infiltration of the site of origin, intravasation, extravasation and invasion of a target organ. All these steps require the degradation of basement membrane components and of extracellular matrix (ECM) macromolecules which is in part mediated by the activity of the matrix metalloproteinases (MMPs). Twenty-four members of the human MMP family have been identified and found to play a crucial role in tumorigenesis by ECM degradation, induction of angiogenesis, inhibition of apoptosis and of the immune system. Extracellular matrix remodelling requires the synergistic action of several metalloproteinases produced by the cancer cells or by the surrounding stromal cells [13]. Based on substrate specificity and domain organisation, MMPs are classified into 5 subgroups: gelatinases, matrilysins, collagenases, stromelysins and membrane bound MMPs. MMP activity is regulated at several levels, including gene expression, secretion, activation and inhibition by their specific endogenous tissue inhibitors TIMPs [5]. Aberrant expression of various members of the MMP family has been associated with a high potential of invasion and metastasis in a broad variety of human carcinomas $[3,7,8,10,21,24]$.

The aim of this study was to explore the expression of a panel of MMPs in primary tumor tissue of 25 breast cancer patients, using quantitative reverse transcriptase polymerase chain reaction (RT-PCR). We focused on MMP2 and MMP9, MMP8 and MMP13, MMP10 and MMP11 as members of the 3 main MMP subclasses: gelatinases, collagenases and stromelysins. We examined whether the RNA expressions of these 6 MMPs are associated with specific clinical and pathological characteristics, including menopausal status, tumor size, tumor grade and lymph node involvement. As we and others have shown an association between individual aberrant MMP expressions and Her2/neu status in breast cancer patients and as distinct breast cancer subtypes have been identified according to their ER, PR and Her2/neu status, we also correlated MMP RNA expression with ER/PR/Her2 profiles of the tumors includ- ed $[2,6,9,15,16,19,20,23]$. Hereby, we focused on the luminal A subtype of invasive ductal carcinomas (IDC) as this subgroup was the best represented in our study. In addition, we investigated plausible co-regulation of MMPs using co-expression analysis.

\section{Materials and methods}

\subsection{Patients}

A total of 25 patients with primary operable breast cancer were included in this study. Patient characteristics, including age at diagnosis and menopausal status, were extracted from clinical files. Tumor size, tumor grade and lymph node status were retrieved from pathology reports, while ER, PR and Her2/neu expression was determined on new immunohistochemical stained sections. Finally, all data were gathered in the central MBC database. All patients were newly diagnosed at the Multidisciplinary Breast Center of the University Hospital of Leuven between 1998 and 2000, underwent mastectomy or local wide excision of their primary breast tumor and axillary lymph node dissection for staging and treatment. None of the patients had received neo-adjuvant treatment, had any history of cancer, multiple tumor foci, bilateral cancer or Paget disease. The presence of formalin-fixed paraffin embedded tumor tissue blocks and freshly frozen tumor tissue stored at $-80^{\circ} \mathrm{C}$ were required for inclusion. This study was conducted in the frame of the EU Framework 6 Cancerdegradome project and its design was approved by the Medical Ethical Committee of the University Hospital Leuven.

\subsection{Pathological assessment of tumor tissue}

All paraffin embedded haematoxylin-eosin (H\&E) slides from the primary tumor as well as from the axillary lymph nodes were reviewed. Tumor typing and grading were performed according to the WHOclassification and the Ellis and Elston grading system respectively. From each frozen block, one $4 \mu \mathrm{m}$ slide was cut and stained with H\&E. Only frozen samples that consist of at least $60 \%$ invasive tumor cells on $\mathrm{H} \& \mathrm{E}$ staining were selected for real time RT-PCR analysis.

Immunohistochemical staining for ER, PR and Her2 was performed on $4 \mu \mathrm{m}$ thick serial paraffin sections. Heat-induced epitope retrieval was carried out in a calibrated water bath $\left(95-99^{\circ} \mathrm{C}\right)$ and antibody complexes were visualised by Envision ${ }^{+}$(Dako, Glostrup, 
Denmark) and DAB. The primary rabbit monoclonal antibodies SP1 directed against ER and SP2 directed against PR (Labvision Corporation, Fremont CA, USA) were applied in a dilution of $1 / 150$ and $1 / 600$ respectively. The primary mouse monoclonal antibody CB11 (Novocastra Laboratories, Newcastle-upon-Tyne, UK) directed against Her2/neu was applied in a dilution of $1 / 40$. Immunostained slides were semi-quantitatively evaluated. Using the Allred score for interpretation of ER and PR immunostaining, the proportion of positive tumor cell nuclei (score 0-5) and the staining intensity (score 0-3) were evaluated. Immunostaining was considered positive when the sum of the proportion and intensity score was $2-8$, while complete absence of staining was defined negative. The FDA-approved DAKO scoring system for Her2/neu immunostaining was applied, taking both the proportion of tumor cells with positive membrane staining and the staining intensity into account. A score of 0 or 1 was defined negative, while a score of 2 or 3 was considered positive for Her2/neu overexpression. Cases with Her2/neu overexpression were further analyzed by dual-colour Fluoresence in situ Hybridization (FISH) in order to distinguish true HER-2/neu gene amplification from polysomy 17 (PathVision, Vysis, Downers Grove, IL, USA). FISH analysis was performed manually on $4 \mu \mathrm{m}$ thick paraffin sections, according to the manufacturer's recommendations. Using a Zeiss Axioplan 2 epifluorescence microscope (Carl Zeiss, Germany) with appropriate filters, signals were counted in 60 nuclei of invasive tumor cells in at least two distinct regions of the tissue section. Tumors showing a mean Her2/chromosome 17 ratio of more than 2 were considered amplified for the Her2 gene, and hence Her2 positive.

\subsection{Cell lines}

Cell lines known to express the investigated MMPs were used as positive controls for real time quantitative RT-PCR. The human breast cancer cell line MDA-MB 231, human PC3 prostate adenocarcinoma cell line and the human G3-61 melanoma cell line were a kind gift of Prof. D. Edwards (University of East Anglia, Norwich UK). All cell lines were cultured in RPMI-1640 medium, supplemented with 10\% Fetal Bovine Serum, $100 \mathrm{units} / \mathrm{ml}$ of penicillin and $100 \mu \mathrm{g} / \mathrm{ml}$ streptomycin and $0.5 \mu \mathrm{g} / \mathrm{ml}$ Fungizone ${ }^{\circledR}$ Antimycotic at $37^{\circ} \mathrm{C}$ and $5 \% \mathrm{CO}_{2}$ (Invitrogen, Carlsbad CA, USA).

\subsection{RNA extraction}

Confluent cell layers were washed twice with phosphate-buffered saline before harvesting. Total RNA was isolated from the cell lines and breast cancer specimens using RNAbee (Campro Scientific, Berlin, Germany) and the SV Total RNA Isolation System (Promega, Madison WI, USA). Approximately $100 \mathrm{mg}$ of fresh frozen tumor tissue was homogenized in $1 \mathrm{ml}$ RNAbee, followed by centrifugation at $14000 \mathrm{rpm}$ for 10 minutes. The clear supernatant was collected into $200 \mu \mathrm{l}$ chloroform, shaken vigorously for 15 seconds, incubated for 3 minutes at room temperature and centrifuged for 15 minutes at $14000 \mathrm{rpm}$. The upper phase was collected into $200 \mu \mathrm{l}$ of $95 \%$ ethanol, mixed and transferred into a spin basket assembly. The SV Total RNA Isolation System protocol was further followed from step 7 to the end, according to the manufacturer's instructions. RNA was resuspended in nuclease free water and concentrations were determined by spectrophotometry using a Nanodrop (Isogen Life Science, IJsselstein, The Netherlands). All RNA samples were stored at $-80^{\circ} \mathrm{C}$ until reverse transcription.

\subsection{Reverse transcription}

In total, $1 \mu \mathrm{g}$ of total RNA was reverse transcribed into cDNA in a $20 \mu \mathrm{l}$ reaction volume containing $2 \mu \mathrm{g}$ random hexamers (GE Healthcare, Buckinghamshire, UK), 200 units of Superscript II reverse transcriptase, 5x first strand buffer (final concentrations $50 \mathrm{mM}$ Tris- $\mathrm{HCl} \mathrm{pH} 8.3 ; 75 \mathrm{mM} \mathrm{KCl} ; 3 \mathrm{mM}$ $\mathrm{MgCl}_{2}, 0.02 \mathrm{mM}$ DTT), $0.5 \mathrm{mM}$ of each deoxynucleotide triphosphate (dATP, dCTP, dGTP, dTTP) and 40 units of RNaseOUT ${ }^{\circledR}$ recombinant ribonuclease inhibitor (Invitrogen, Carlsbad CA, USA). Reverse transcription was carried out at $70^{\circ} \mathrm{C}$ for 10 minutes and continued at $42^{\circ} \mathrm{C}$ for 1 hour. cDNA was stored at $-80^{\circ} \mathrm{C}$ until use.

\subsection{Quantitative RT-PCR}

Specific primers and fluorogenic probes for MMP2, MMP8, MMP9, MMP10, MMP11 and MMP13 were designed with Primer Express software (Applied Biosystems, Foster City CA, USA) and synthesized by Eurogentec (Eurogentec, Seraing, Belgium). The sequences for the MMP primers and probes are given in Table 1. In order to prevent amplification of genomic DNA, primers were generated with sequences within different exons, close to intron-exon boundaries. PCR 
Table 1

TaqMan primer and probe sequences for the studied human MMPs

\begin{tabular}{|c|c|c|c|c|}
\hline Gene & $\begin{array}{l}\text { Genebank } \\
\text { accesion no. }\end{array}$ & sequence of primers pairs and FAM-TAMRA probe* & exon boundary & $\begin{array}{l}\text { amplicon } \\
\text { size (bp) }\end{array}$ \\
\hline MMP2 & NM004530 & $\begin{array}{l}\text { TGGCGATGGATACCCCTTT } \\
\text { TTCTCCCAAGGTCCATAGCTCAT } \\
\text { CTCCTGGCTCATGCCTTCGCCC }\end{array}$ & exon12/exon13 & 83 \\
\hline MMP9 & NM004994 & $\begin{array}{l}\text { CCTGGGCAGATTCCAAACCT } \\
\text { GCAAGTCTTCCGAGTAGTTTTGGAT } \\
\text { CTCAAGTGGCACCACCACAACATCACC }\end{array}$ & exon $12 /$ exon 13 & 54 \\
\hline MMP8 & NM002424 & $\begin{array}{l}\text { CACTCCCTCAAGATGACATCGA } \\
\text { ACGGAGTGTGGTGATAGCATCA } \\
\text { CAAGCAACCCTATCCAACCTACTGGACCAA }\end{array}$ & exon2/exon 3 & 120 \\
\hline MMP13 & NM002427 & $\begin{array}{l}\text { AAATTATGGAGGAGATGCCCATT } \\
\text { TCCTTGGAGTGGTCAAGACCTAA } \\
\text { CTACAACTTGTTTCTTGTTGCTGCGCATGA }\end{array}$ & exon5/exon6 & 91 \\
\hline MMP10 & NM002425 & $\begin{array}{l}\text { GGACCTGGGCTTTATGGAGATAT } \\
\text { CCCAGGGAGTGGCCAAGT } \\
\text { CATCAGGCACCAATTTATTCCTCGTTGCT }\end{array}$ & exon $9 /$ exon 10 & 82 \\
\hline MMP11 & NM005940 & $\begin{array}{l}\text { CCGCCAGATGCCTGTGA } \\
\text { CGGAGGCGCCACACAA } \\
\text { CCTCCTTTGACGCGGTCTCCACC }\end{array}$ & & 66 \\
\hline
\end{tabular}

reactions were carried out on the ABI Prism 7700 Sequence Detection System (Applied Biosystems, Foster City CA, USA). Each reaction was performed in a $25 \mu \mathrm{l}$ reaction volume, containing $5 \mathrm{ng}$ cDNA (1 $\mathrm{ng}$ for $18 \mathrm{~S}$ analyses), $8.33 \mu 1 \mathrm{qPCR}$ Master mix (Eurogentec, Seraing, Belgium), $100 \mathrm{nM}$ probe and $200 \mathrm{nM}$ each primer. Conditions for the PCR reaction were 2 min at $50^{\circ} \mathrm{C}, 10 \mathrm{~min}$ at $95^{\circ} \mathrm{C}$ followed by 40 cycles of $15 \mathrm{~s}$ at $95^{\circ} \mathrm{C}$ and $1 \mathrm{~min}$ at $60^{\circ} \mathrm{C}$. The $18 \mathrm{~S}$ ribosomal RNA (18S rRNA) gene was used as an endogenous control to normalize for differences in the amount of total RNA in each sample and its expression was determined with a commercially available 18S rRNA primer and probe set (Applied Biosystems, Foster City CA, USA). To determine the relative RNA levels within the samples, standard curves for the PCR reactions were prepared. Serial cDNA dilutions of the human breast cancer cell line MDA-MB 231 served as positive control for $18 \mathrm{~S}$ rRNA, while dilution series of the human PC3 prostate adenocarcinoma cell line and of the human G3-61 melanoma cell line served as positive controls for MMP2, MMP9, MMP10, MMP11, MMP13 and for MMP8 respectively.

\subsection{Statistical analyses}

Assumption of normality was verified using the normal probability plot, Shapiro-Wilk's W test and the Levene's test for homogeneity of variances. None of the data were found to have a normal distribution. The
MMP RNA expression was defined high when it was above the median expression of this specific MMP. Associations between MMP expression (high/low) and various clinicopathological variables were determined using the two-tailed Fisher's exact test. Associations among the individual MMP normalized values were evaluated by the Spearman's Rank correlation coefficient. Statistical analyses were performed using the software package SPSS version 13, the level of significance being set at $p \leqslant 0.05$.

\section{Results}

\subsection{Patient and tumor characteristics}

The median age at diagnosis of all patients was 51 (range 36-79). Twelve (48\%) patients were premenopausal with a median age of 47 (range 36-51) and $13(52 \%)$ were postmenopausal with a median age of 65 (range 51-79). Small and large tumors were equally distributed, 12 (48\%) and 13 (52\%) tumors respectively. None of the tumors were well differentiated, $14(56 \%)$ were moderately and $11(44 \%)$ were poorly differentiated. No nodal involvement was found in 7 (28\%) patients whereas the remaining 18 (72\%) were lymph node positive. Stratification of tumors into distinct subtypes according to ER, PR and Her2/neu status resulted in 19 luminal A invasive ductal carcinomas of no specific type, 2 basal-like carcinomas, 1 Her2+/ER- 
tumor, 1 luminal A invasive lobular carcinoma of the pleomorphic type and 2 luminal A invasive lobular carcinomas of the classic type. According to the AJCC staging system, 4 patients had stage I disease, 13 had stage II disease and 8 had stage III disease.

\subsection{Association of MMP expressions and clinicopathological parameters}

Table 2 summarizes the most important clinical and pathological characteristics at the time of diagnosis; including menopausal status, tumor size, tumor grade and lymph node involvement. No difference was found in the RNA expression levels of MMP2, MMP9, MMP8, MMP13, MMP10 and MMP11 between small and large tumors, moderately and poorly differentiated tumors or between tumors with or without lymph node involvement. MMP2 expression was significantly correlated with menopausal status ( $p=0.02$ ): $75 \%$ of tumors from premenopausal women showed high MMP2 RNA levels, while this was only the case in $23 \%$ of tumors from postmenopausal women. As the luminal A subgroup of invasive ductal carcinomas was the only breast cancer subtype that was well represented in our study population (19 of 25 tumors), we further investigated the MMP expression profiles in these tumors (Table 3). Within this group of tumors the association between MMP2 RNA expression and menopausal status was even more significant $(p=0.005)$ than in the whole study population with $80 \%$ of premenopausal patients and only $11 \%$ of postmenopausal women expressing high MMP2 levels. Furthermore, we found that in this particular tumor subtype also MMP11 RNA expression was correlated with menopausal status $(p=0.02)$.

\subsection{MMP co-expression analyses}

The present real time RT-PCR data were also assessed by co-expression analyses to look for potential patterns of MMP co-regulation in breast cancer (Table 4). These analyses revealed that 2 particular MMP gene pairs showed a weak association of their expression: MMP2 and MMP10 on the one hand, and MMP8 and MMP9 on the other. MMP11 and MMP13 RNA expressions were significantly correlated with both sets of genes.

\section{Discussion}

This report presents an expression profile analysis of a panel of 6 MMPs on the RNA level in 25 primary invasive breast carcinomas. Using real time RTPCR, we examined the RNA expressions of the gelatinases MMP2 and MMP9, the collagenases MMP8 and MMP13 and the stromelysins MMP10 and MMP11, correlated our findings with classical clinicopathological parameters and explored co-expression patterns of MMP gene pairs. Within the whole study group we found significantly higher MMP2 RNA levels in tumors from premenopausal patients than in tumors from postmenopausal patients. However, this finding is most probably the reflection of the result found in luminal A IDCs as these tumors represent 19 out of 25 tumors in our study and show the same result at an even higher level of significance. Moreover, these tumors also showed significantly higher MMP11 expression levels in premenopausal than in postmenopausal women. No significant associations were found between the RNA expressions of the other MMPs (MMP8, MMP9, MMP10 and MMP13) and menopausal status, neither for the whole group, nor for the subgroup of luminal A IDC. None of the 6 MMPs studied were differentially expressed according to tumor size, tumor grade or lymph node involvement.

As mainly conflicting data have been reported on correlations between individual MMP RNA expressions and clinicopathological parameters, our findings are consistent with some reports and discordant with others $[4,11,12,14,20,22]$. This discrepancy may be partly explained by the use of different patient selection criteria or by the different analytical sensitivities of the methods used, including in situ hybridisation, northern blotting and quantitative RT-PCR. Therefore, prudence is called for comparison of studies using various technologies.

Pair-wise expression analyses of the present data indicated a potential co-regulation of two sets of gene pairs: MMP2 and MMP10 on the one hand and MMP8 and MMP9 on the other. Hence, further investigation of the underlying signalling pathways of MMP regulation seems indicated.

In conclusion, we observed higher MMP2 RNA expression levels in tumors from premenopausal patients than in those from postmenopausal patients. As luminal A invasive ductal carcinomas represent 19 out of 25 tumors in our study, this result was a reflection of the same association found in this subtype. Moreover, differential expression of MMP2 in relation to 

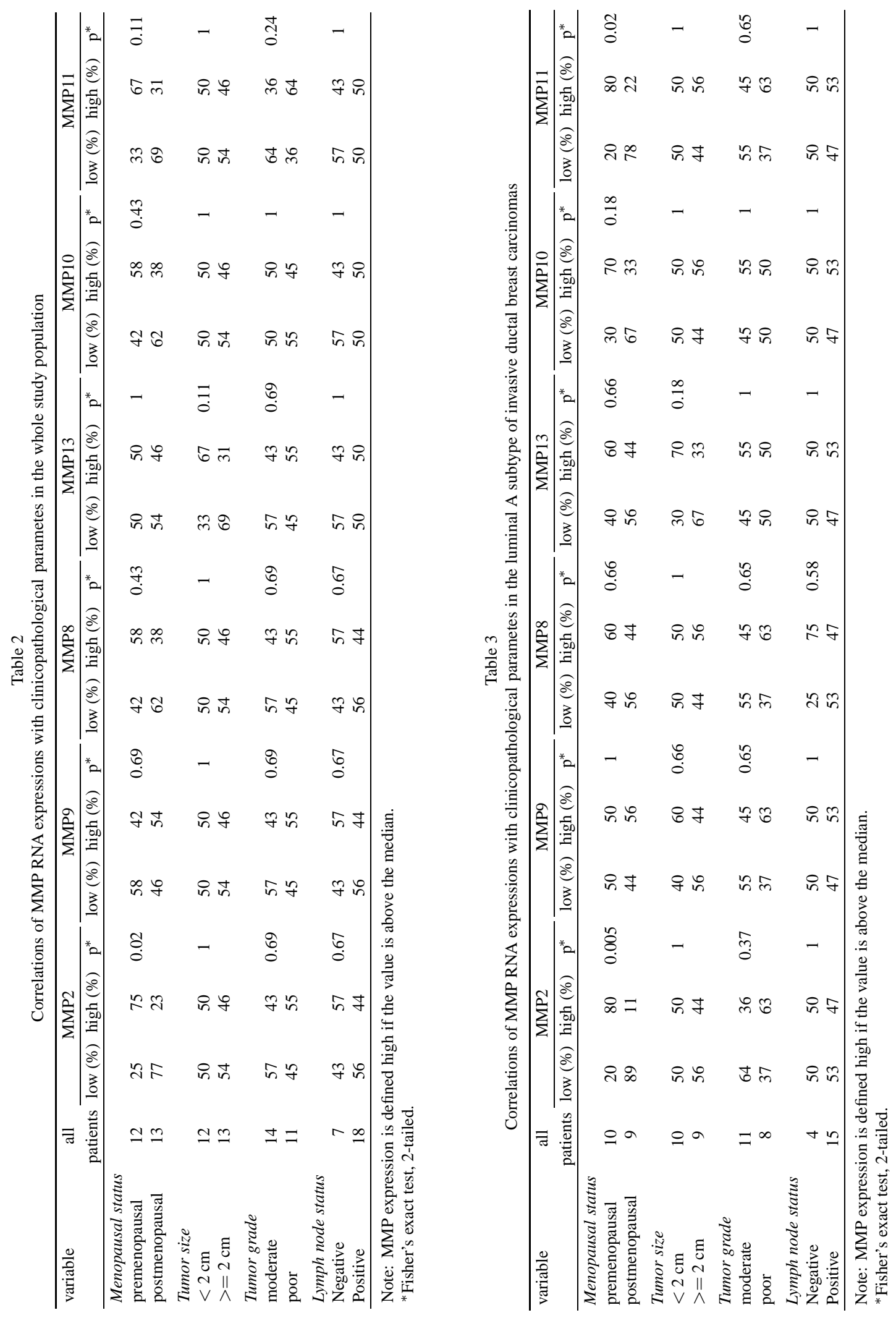
Table 4

Correlation table comparing individual MMP RNA expressions

\begin{tabular}{llllll}
\hline & MMP2 & MMP9 & MMP8 & MMP13 & MMP10 \\
\hline MMP2 & $r s=1$ & & & & \\
MMP9 & $p=0.64$ & $r s=1$ & & & \\
& $r s=0.10$ & & & & \\
MMP8 & $p=0.24$ & $p=0.03$ & $r s=1$ & & \\
& $r s=0.24$ & $r s=0.43$ & & & \\
MMP13 & $p=0.012$ & $p=0.0009$ & $p=0.05$ & $r s=1$ & \\
& $r s=0.49$ & $r s=0.62$ & $r s=0.39$ & & \\
MMP10 & $p=0.019$ & $p=0.18$ & $p=0.68$ & $p=0.0061$ & $r s=1$ \\
& $r s=0.47$ & $r s=0.28$ & $r s=0.09$ & $r s=0.54$ & \\
MMP11 & $p=0.002$ & $p=0.04$ & $p=0.28$ & $p=0.0003$ & $p=0.002$ \\
& $r s=0.58$ & $r s=0.42$ & $r s=0.22$ & $r s=0.73$ & $r s=0.58$ \\
\hline
\end{tabular}

rs $=$ Spearman Rank Correlation coefficient. Values in bold indicate $p<0.05$

note: all correlations are positive, defined weak if $0<r s<0.5$ and strong if $0.5<r s<1.0$.

menopausal status was found at an even higher level of significance. Furthermore, within this subgroup MMP11 RNA expression levels were also significantly higher in premenopausal women as compared to postmenopausal women. Finally, our data suggest coregulation of MMP2 and MMP10 on the one hand and of MMP8 and MMP9 on the other. We plan to extend our study of MMP expression profiles to a larger set of invasive breast cancers with a sufficient number of cases in 4 main groups: luminal A, luminal B, basal-like and $\mathrm{Her} 2^{+} / \mathrm{ER}^{-}$subtype.

\section{Acknowledgements}

This work was supported by the "Vlaamse Liga tegen Kanker" and EU Framework Programme 6 (grant number LSHC-CT-2003-503297). We would like to thank Prof. Edwards and Dr. Pennington for the kind gift of the PC3 and G3-61 cell lines.

\section{References}

[1] L.A. Carey, C.M. Perou, C.A. Livasy, L.G. Dressler, D. Cowan, K. Conway, G. Karaca, M.A. Troester, C.K. Tse, S. Edmiston, S.L. Deming, J. Geradts, M.C. Cheang, T.O. Nielsen, P.G. Moorman, H.S. Earp and R.C. Millikan, Race, breast cancer subtypes, and survival in the Carolina Breast Cancer Study, JAMA 295 (2006), 2492-2502.

[2] J. Decock, W. Hendrickx, H. Wildiers, M.R. Christiaens, P. Neven, M. Drijkoningen and R. Paridaens, Plasma gelatinase levels in patients with primary breast cancer in relation to axillary lymph node status, Her2/neu expression and other clinicopathological variables, Clin Exp Metastasis 22 (2005), 495-502.

[3] M.J. Duffy, T.M. Maguire, A. Hill, E. McDermott and N. O'Higgins, Metalloproteinases: role in breast carcinogenesis, invasion and metastasis, Breast Cancer Res 2 (2000), 252257.
[4] S.Q. Fan, Q.Y. Wei, M.R. Li, L.Q. Zhang and Q.C. Liang, Expression and clinical significance of MMP-2, MMP-9,TIMP1, and TIMP-2 in breast carcinoma, Ai Zheng 22 (2003), 968973.

[5] A.R. Folgueras, A.M. Pendas, L.M. Sanchez and C. LopezOtin, Matrix metalloproteinases in cancer: from new functions to improved inhibition strategies, Int J Dev Biol 48 (2004), $411-424$.

[6] D. Giunciuglio, M. Culty, G. Fassina, L. Masiello, A. Melchiori, G. Paglialunga, G. Arand, F. Ciardiello, F. Basolo and E.W. Thompson, Invasive phenotype of MCF10A cells overexpressing c-Ha-ras and c-erbB-2 oncogenes, Int J Cancer 63 (1995), 815-822.

[7] M.M. Gueders, J.M. Foidart, A. Noel and D.D. Cataldo, Matrix metalloproteinases (MMPs) and tissue inhibitors of MMPs in the respiratory tract: potential implications in asthma and other lung diseases, Eur J Pharmacol 533 (2006), 133-144.

[8] S.K. Katiyar, Matrix metalloproteinases in cancer metastasis: molecular targets for prostate cancer prevention by green tea polyphenols and grape seed proanthocyanidins, Endocr Metab Immune Disord Drug Targets 6 (2006), 17-24.

[9] G. La Rocca, I. Pucci-Minafra, A. Marrazzo, P. Taormina and S. Minafra, Zymographic detection and clinical correlations of MMP-2 and MMP-9 in breast cancer sera, Br J Cancer $\mathbf{9 0}$ (2004), 1414-1421.

[10] R. la-aho and V.M. Kahari, Collagenases in cancer, Biochimie 87 (2005), 273-286.

[11] A. Lebeau, C. Muller-Aufdemkamp, C. Allmacher, U. Sauer, A. Nerlich, R. Lichtinghagen and U. Lohrs, Cellular protein and mRNA expression patterns of matrix metalloproteinases2, -3 and -9 in human breast cancer: correlation with tumour growth, J Mol Histol 35 (2004), 443-455.

[12] A.S. Mellick, D. Blackmore, S.R. Weinstein and L.R. Griffiths, An assessment of MMP and TIMP gene expression in cell lines and stroma - tumour differences in microdissected breast cancer biopsies, Tumour Biol 24 (2003), 258-270.

[13] M.M. Mueller and N.E. Fusenig, Friends or foes - bipolar effects of the tumour stroma in cancer, Nat Rev Cancer 4 (2004), 839-849.

[14] M.M. Pacheco, M. Mourao, E.B. Mantovani, I.N. Nishimoto and M.M. Brentani, Expression of gelatinases A and B, stromelysin-3 and matrilysin genes in breast carcinomas: clinico-pathological correlations, Clin Exp Metastasis 16 (1998), 577-585. 
[15] J.M. Pellikainen, K.M. Ropponen, V.V. Kataja, J.K. Kellokoski, M.J. Eskelinen and V.M. Kosma, Expression of matrix metalloproteinase (MMP)-2 and MMP-9 in breast cancer with a special reference to activator protein-2, HER2, and prognosis, Clin Cancer Res 10 (2004), 7621-7628.

[16] A. Roetger, A. Merschjann, T. Dittmar, C. Jackisch, A. Barnekow and B. Brandt, Selection of potentially metastatic subpopulations expressing c-erbB-2 from breast cancer tissue by use of an extravasation model, Am J Pathol 153 (1998), 1797-1806.

[17] T. Sorlie, C.M. Perou, R. Tibshirani, T. Aas, S. Geisler, H. Johnsen, T. Hastie, M.B. Eisen, R.M. van de, S.S. Jeffrey, T. Thorsen, H. Quist, J.C. Matese, P.O. Brown, D. Botstein, L.P. Eystein and A.L. Borresen-Dale, Gene expression patterns of breast carcinomas distinguish tumor subclasses with clinical implications, Proc Natl Acad Sci USA 98 (2001), 1086910874.

[18] T. Sorlie, R. Tibshirani, J. Parker, T. Hastie, J.S. Marron, A. Nobel, S. Deng, H. Johnsen, R. Pesich, S. Geisler, J. Demeter, C.M. Perou, P.E. Lonning, P.O. Brown, A.L. BorresenDale and D. Botstein, Repeated observation of breast tumor subtypes in independent gene expression data sets, Proc Natl Acad Sci USA 100 (2003), 8418-8423.
[19] M. Tan, J. Yao and D. Yu, Overexpression of the c-erbB-2 gene enhanced intrinsic metastasis potential in human breast cancer cells without increasing their transformation abilities, Cancer Res 57 (1997), 1199-1205.

[20] B. Tetu, J. Brisson, H. Lapointe and P. Bernard, Prognostic significance of stromelysin 3, gelatinase A, and urokinase expression in breast cancer, Hum Pathol 29 (1998), 979-985.

[21] T. Turpeenniemi-Hujanen, Gelatinases (MMP-2 and -9) and their natural inhibitors as prognostic indicators in solid cancers, Biochimie 87 (2005), 287-297.

[22] C.H. Xiao, Y.M. Feng, X.Q. Li and X.S. Hao, Clinical significance and relationship of expression of matrix metalloproteinase-2 mRNA with breast cancer metastasis, Zhonghua Wai Ke Za Zhi 43 (2005), 599-604.

[23] F.J. Xu, S. Stack, C. Boyer, K. O’Briant, R. Whitaker, G.B. Mills, Y.H. Yu and R.C. Bast, Jr., Heregulin and agonistic antip185(c-erbB2) antibodies inhibit proliferation but increase invasiveness of breast cancer cells that overexpress p185(cerbB2): increased invasiveness may contribute to poor prognosis, Clin Cancer Res 3 (1997), 1629-1634.

[24] S. Zucker and J. Vacirca, Role of matrix metalloproteinases (MMPs) in colorectal cancer, Cancer Metastasis Rev 23 (2004), 101-117. 


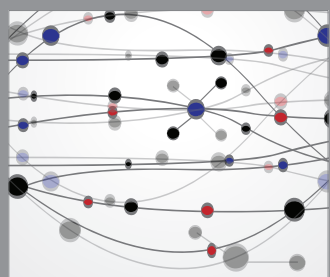

The Scientific World Journal
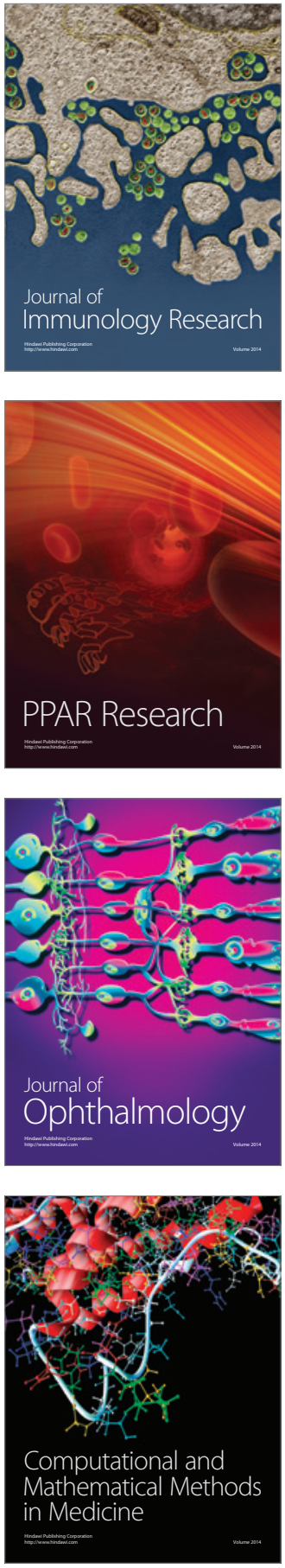

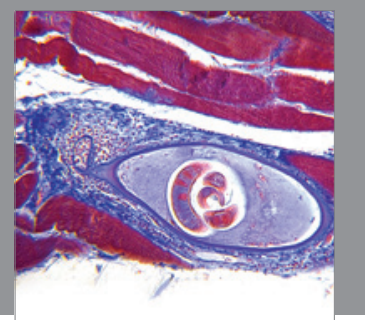

Gastroenterology

Research and Practice
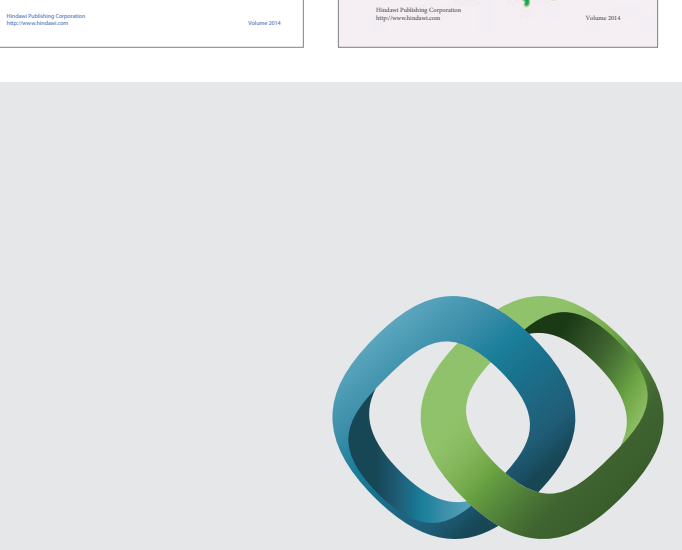

\section{Hindawi}

Submit your manuscripts at

http://www.hindawi.com
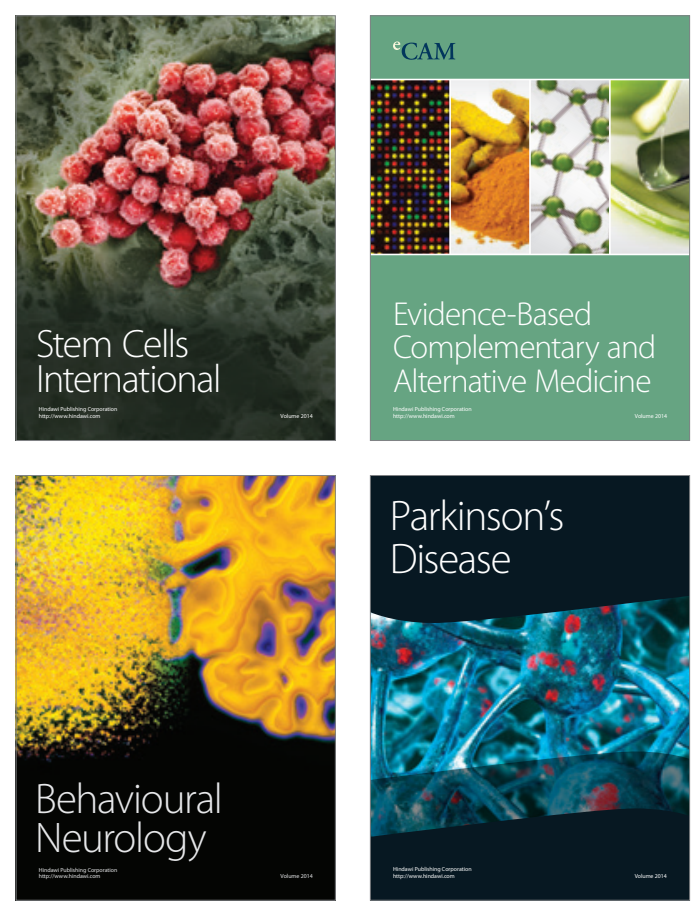

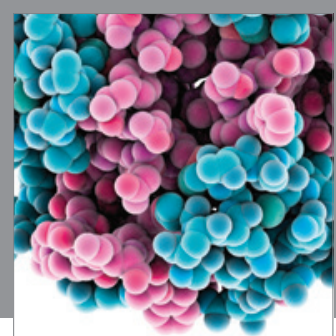

Journal of
Diabetes Research

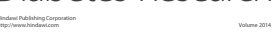

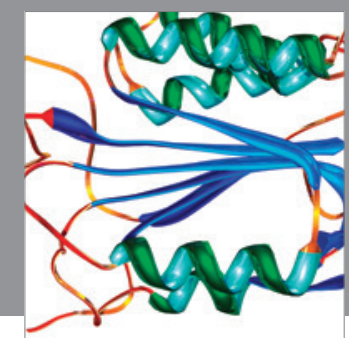

Disease Markers
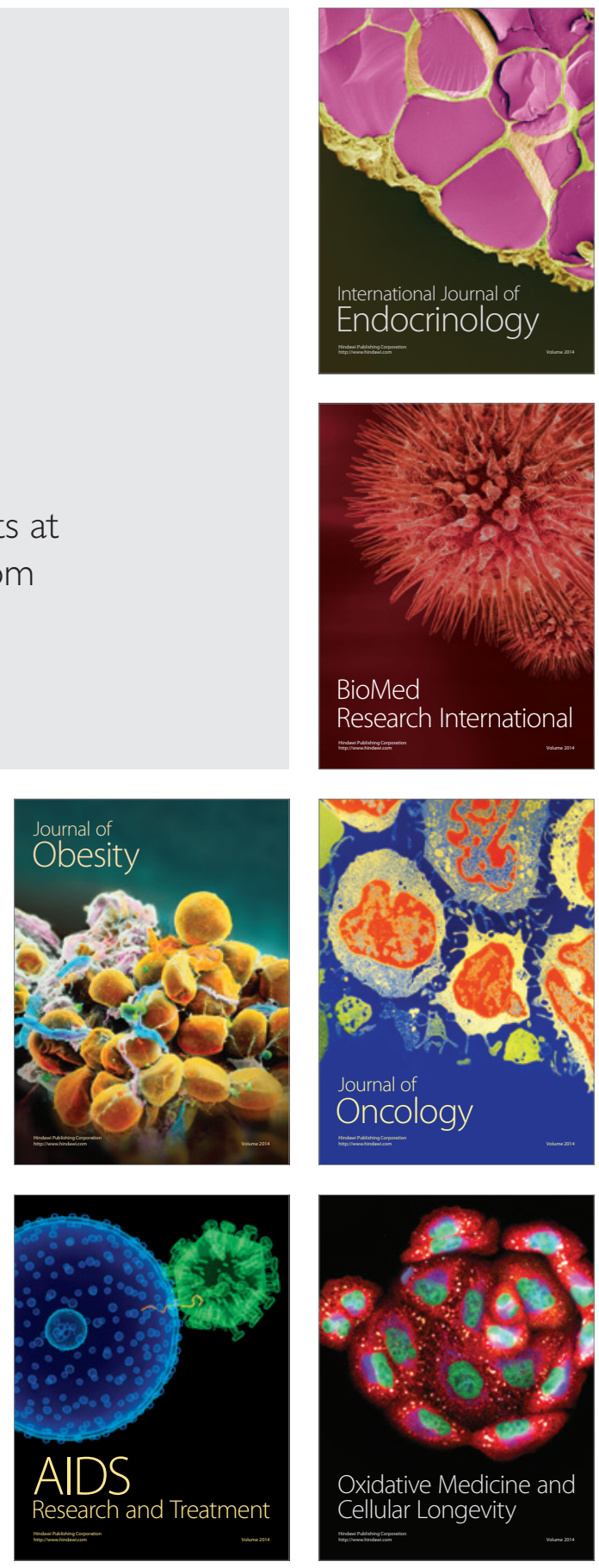